\title{
el Proteinograma EN LA toxicosis
}

\author{
DTS. GUILLERMO BARDALES * y RAMON MONTERO
}

Cátedta de Pediatría del Prof. Aníbal Ariztía.

Hospital "Luis Calvo Mackenna". Santiago.

Este trabajo tuyo por objeto corroborar en nuestro medio las interesantes investigaciones electroforéticas realizadas en niños distróficos con toxicosis por la Clínica Infantil del Prof. Hungerland en Giessen, Alemania 1. Hungerland, estudiando los resultados obtenidos con la seroelectroforesis en papal en niños con toxicosis después de la hidratación, pudo comprobar una significativa alza de los valores relativos de las gamas globulinas. Estas alteraciones, en cambio, no pudieron apreciarlas en cuadros eméticos sin síndrome tóxico, como sucede en la píloro estenosis del lactante menor ${ }^{\mathbf{1 . 2}}$.

Para llevar a efecto nuestro trabajo realizamos una serie de determinaciones electroforéticas en el suero de lactantes distróficos con toxicosis, especialmente en el momento de su ingreso al Hospital, e inmediatamente después de terminada su hidratación con la flebaclisis gluco-salina.

\section{MATERIAL Y MÉTODO}

Las determinaciones electroforéticas se realizaron en el suero de 20 lactantes distróficos con toxicosis y de ambos sexos, antes y después de su hidratación con fleboclisis glueosalina (S. Ringer con S. Glucosado al $5 \%$, en partes iguales). Sus edades fluctuaron entre los 2 y 24 meses;

- Becario peruano. el peso osciló entre los 2.700 y los 8.000 gr. Todos fueron atendidos en el Servicio de Media Hospitalización del Hospital "Luis Calvo Mackenna" durante los meses de Diciembre de 1957 y Enero de 1958.

Para determinar el proteínograma hemos empleado el conocido método de Grassmann-Kendel-Hannig de electroforesis en papel, con equipo Elphor H. ${ }^{3.4}$. Las proteínas totales se determinaron con el método químico del Biuret ${ }^{3}$.

\section{RESULTADOS}

A] comparar los valores absolutos encontrados en nuestros 20 casos, antes y después de la fleboclisis, observamos cifras bajas en estos últimos valores que son significativos para la seroalbúmina (5.32); globulina alfa 1 (4.25); globulina alfa $2(2.81)$ y globulina beta (5.17). Esta baja no afectó a la gama globulina (0.12). Cuadro $\mathrm{N}^{\circ} 2$.

En cuanto a sus valores relativos hubo 15 casos que mostraron alza especialmente significativas con respecto al valor de sus gamas globulinas después de la hidratación, no observándose este hecho en 5 casos; sin embargo, es de advertir que estas últimas no logramon modificar la cifra total de valores relativos. Se obtuvo $\mathrm{X}$

un _-_ de 3.42 , que por ser superior a sigma

2 resultó de significación estadística.

COADRO NQ 1

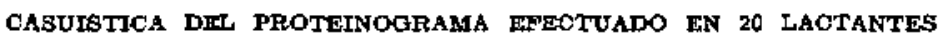
DISTEOFICOS CON TOXICOSIS

\begin{tabular}{|c|c|c|c|c|}
\hline & $\boldsymbol{E} a \mathrm{a}$ & I d a & Edad & Tot a \\
\hline Sexo: & 2-6 meses & 7-12 meses & 13-24 meses & \\
\hline $\begin{array}{c}\text { Maseulino . . . . } \\
\text { Peso. . . . . }\end{array}$ & $\begin{array}{c}5 \\
3.400-4.200 \mathrm{gr} .\end{array}$ & $\stackrel{5}{4.200-7.200 \mathrm{gr}}$ & $\begin{array}{c}2 \\
4.550-8.650 \mathrm{gr}\end{array}$ & 12 \\
\hline $\begin{array}{l}\text { Fementno . . : } \\
\text { Peso . . : }\end{array}$ & $\stackrel{3}{2.700-4.100} \mathrm{gr}$. & $\frac{1}{4.620 \mathrm{gr} .}$ & $\stackrel{4}{5.700-8 \mathrm{~kg}}$ & 8 \\
\hline TOTAL ..... & B & 6 & 6 & 20 \\
\hline
\end{tabular}


CDADRO NO 2

\begin{abstract}
PROMEDTO DE LOS "VALORTAS ABSOLUTOS" $Y$ DE LAS DESVLACIONES STANDARD DEL PROTEINOGRAMA EN 20 LACTANTES DISTROFICOS CON Toxicosis (2 a 24 meses de edad)
\end{abstract}

COMPARADO CON HL PROMEDIO NORMAL DF VALORES ABSOLUTOS

\begin{tabular}{|c|c|c|c|c|}
\hline Promedlo or & Tolores normoles & Antes fleboclisls & Despues fleboclista & Colc ste slantyla \\
\hline 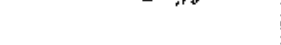 & & Velores encontrados & Volores encontradce & \\
\hline Seroalbúmine . . & $40^{\prime} 15 \pm 3,34$ & $40^{\prime} .0 \pm 4^{\prime} 78$ & $32^{\prime} 22 \pm 4^{+54}$ & $5 \cdot 32(+)$ \\
\hline $\begin{array}{l}\text { Eeroglobulina: } \\
\text { Alfa } 1 . . .\end{array}$ & $3.03 \pm 0.43$ & $4^{\prime 39} \pm 0^{\prime} 88$ & $320 \pm 0,96$ & $425(+)$ \\
\hline $\begin{array}{l}\text { Seroglobulina: } \\
\text { Alfa } 2 .\end{array}$ & $9^{1} 56 \pm 1^{1} 05$ & $10^{\prime} 68 \pm 1^{\prime} 74$ & $9^{\prime} 02 \pm 206$ & $2^{\prime} 81(+)$ \\
\hline $\begin{array}{l}\text { Beroglobulina: } \\
\text { Beta....... }\end{array}$ & $8^{\prime} 21 \pm 1^{\prime} 05$ & $10^{\prime} 23 \pm 1 \cdot 63$ & $7^{\prime} 65 \pm 1 ' 35$ & $5 \cdot 17(+)$ \\
\hline $\begin{array}{l}\text { Beroglobuliza: } \\
\text { Gama . . . . . }\end{array}$ & $6 ' 58 \pm 113$ & $B^{\prime} 98 \pm 2^{\prime} 16$ & $9^{\circ} 06 \pm I^{* 85}$ & $0^{\prime} 12$ \\
\hline $\begin{array}{l}\text { Frot. Total . . } \\
\text { (MÁt. Quím.) }\end{array}$ & $60^{\prime} 65 \pm 4^{\prime} 59$ & $74^{2} 22 \pm 8^{\prime} 64$ & $61^{\prime} 43 \pm \theta^{\prime} 52$ & $473(+)$ \\
\hline
\end{tabular}

Con respecto a las proteinas totales, se observó una baja significativa (4.73) después de la fleboclisis. (Cuadro $N^{\circ} 3$ ).

\section{COMENTARIO}

De las observaciones realizadas en el estudio electroforético de 20 lactantes distróficos con toxicosis, antes y después de la hidratación con fleboclisis glucosalina, hemos encontrado modificaciones significativas en el proteínograma y que en general están de acuerdo con lo publicado por los autores alemanes ${ }^{1-2}$, o sea, se aprecia un aumento relativo de las gamas globulinas después de la hidratación.

Es de advertir también el hecho que observamos dentro de los valores absolutos y relativos de las gamas globulinas del distrófico con toxicosis cifras más al-

COADRO No 3

PROMEDTO DE IOS "VALORES RELATIVOS" $Y$ DI LAg DBBVIACIONES STANDARD DEL PROTEMNOGRAMA EN 20 LACTANTES DIBTROFICOS CON TOXICOSIS ( 2 a 24 mereg de edad)

COMPARADO CON EL PROMERDIO NORMAL DER VALORES RELATIVOS

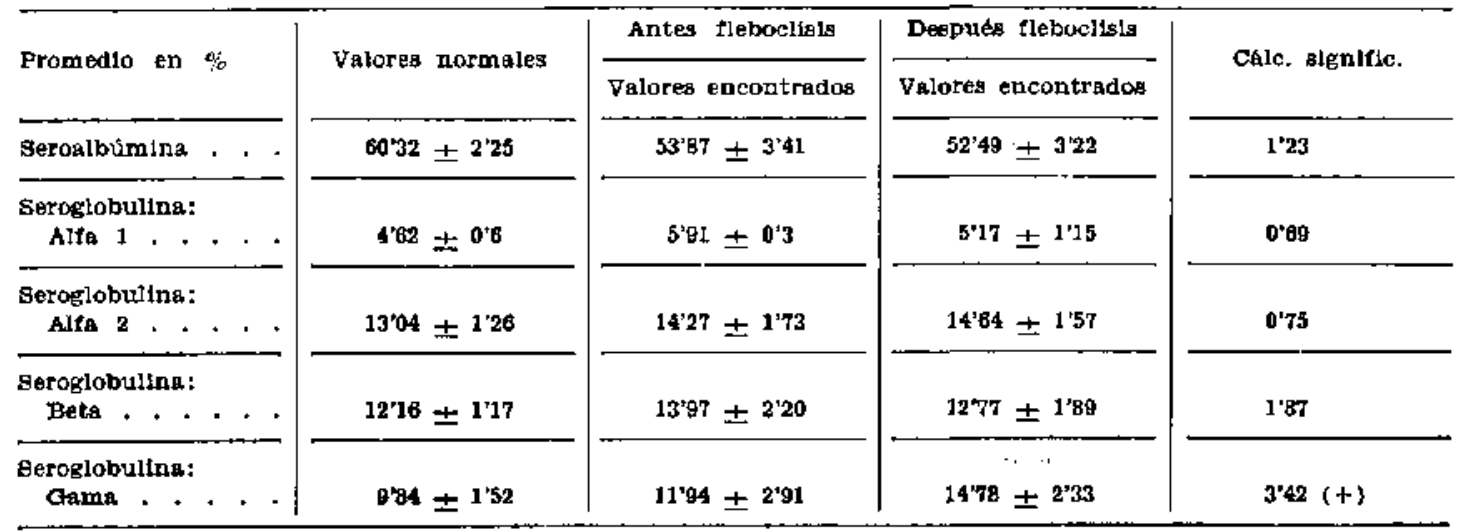


tas que los de niños normales, para lo cual hemos comparado estos valores con una tabla correspondiente a niños sanos realizado en nuestro medio y que figura adjunta al cuadro 2 y 3 . Bollo y Montero ${ }^{4}$ encontraron estas mismas alteraciones cuando realizaron su trabajo de "Distrofia con edemas", y Io explican como una probable compensación de las globulinas extra-hepáticas para impedir la hipoproteinemias, en otras palabras, como un esfuerzo para mantener el valor oncónico del plasma ante el severo déficit de serinas de dicha distrofia con esteatosis hepática, como ha sido demostrado por Gómez "; Scroggie "; Meneghello ${ }^{t}$ y otros.

\section{RESUMEN}

Los autores estudian el proteínograma mediante la electroforesis en papel de 20 lactantes distróficos con toxicosis. antes y después de la hidratación con fleboclisis glucosalina.

En sus resultados encontraron una significativa baja de las proteínas totales después de la hidratación, comparadas con cifras previas a la fleboclisis y con valores de niños normales de nuestro medio. Además hubo una significativa baja de los valores absolutos después de la hidratación en la seroalbúmina, como en las globulinas alfa, uno y dos; y en la globulina beta. sin alterar mayormente a la gama globulina.

En cuanto a los valores relativos, observan después de la hidratación una alza significativa de las gamas globulinas, alza que no es acompañada por las otras fracciones proteicas.

Los autores consideran que este último fenómeno podría explicarse por una acción compensadora selectiva del sistema retículo-endotelial o linfático, lugar de origen de las gama globulinas, destinado a evitar la hemodilución.

\section{SUMMARY}

\section{PROTELNOGRAM IN TOXICOSIS.}

The authors study the electrophoretic pattern in 20 infants with malnutrition and toxicosis, before and after the paraenteral hidratation.

They observed a significative decrease in the absolute values of the seroalbumine, as well as the alpha 1 , alpha 2 , and beta globulins after hidratation, with no change in the gamma globulins. There was a significative rise in the relative values of the gamma globulin after hidratation.

The authors considered that the relative high values of the gamma globulins can be explained as a regulative selective measure of the reticuloendotelial or lymphatic systems in order to avoid hemodilution.

\section{BIBLIOGRAFÍA}

1.-SCHMWT, G. W. - Ueber das Verhalten des gerumeiwelssgehaltes im ersten Lebensjaht. Zettschrlft Kinderhellkunde. $71: 483$ (1952).

2.-KOERVER, H, A. MASSENBERG und L, SCHOMER. Ueber Ursachen und Bedeutung clines ernledrlgten Globulinsplegels in Blutserum bel Sauplisen. Monatsenrift Kinderbellkunde. 100:230 (I052)

3.-MAsJUAN, F. - "Microelectrofores's en Papel de las seroproténas en NIROs Sanos de 4 a 30 meses de edad". Tesis 1957.

4.-BOLLO, F. y R. MON THRO. -- "Electroprotidograma en la Distrofla con Edemas". - Rev. Chll. de Ped, 1:6, 1957.

5.-GOMgz, F. g cois, - "Fstudios en niflos desnutridog". - American Journal of Dlseases of Children. 87:673, 1954 .

o. SCROGGIE, A. - "Trastornos nutritivos crónicos y sus aspectos médico-sociales". Rev. Chil. Ped. 19:1, 1948.

7.-MENEGEELLO, J. - "Evoluclón Intrahospitalarla del lactante distrofico menor de 1 año". Rev. Chll. Ped. 23:3, 1952.

8.-RIVA, G. DAs Serumeltrefsbula. Hans \#uber Vellig, 1957, Bern und gtutteart.

9.-ANTWETLER, $H$. $G$, - Die Quantitatlve Elekirophorese tu der Medizin. $2^{\mathrm{a}}$ Autlage, Springer Verlas, 1957. Berlín-Goettingen-Ifejäelberg. 